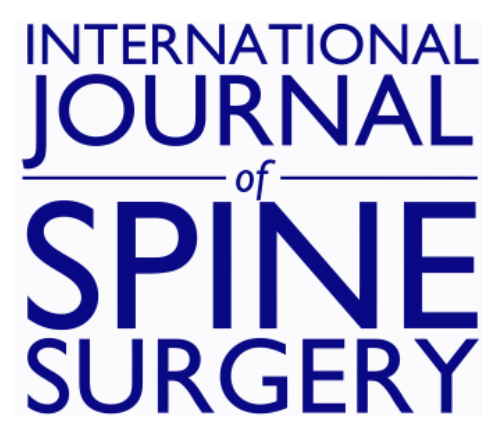

\title{
Ceftriaxone Plus Methylprednisolone Combination Therapy Versus Methylprednisolone Monotherapy in Patients With Acute Spinal Cord Injury: A Randomized, Triple-Blind Clinical Trial
}

FARHAD MIRZAEI, ALI MESHKINI, BOHLOOL HABIBI, FIROOZ SALEHPOUR, EBRAHIM RAFEI, WOURIA FATHI, SEYED HAMED NASERI ALAVI, ALIREZA MAJDI, SEPIDEH RAHIGH AGHASAN and SEYED AHMAD NASERI ALAVI

Int J Spine Surg 2020, 14 (5) 706-712

doi: https://doi.org/10.14444/7102

http://ijssurgery.com/content/14/5/706

This information is current as of April 26, 2023.

Email Alerts Receive free email-alerts when new articles cite this article. Sign up at: http://ijssurgery.com/alerts 


\title{
Ceftriaxone Plus Methylprednisolone Combination Therapy Versus Methylprednisolone Monotherapy in Patients With Acute Spinal Cord Injury: A Randomized, Triple-Blind Clinical Trial
}

\author{
FARHAD MIRZAEI, MD,${ }^{1}$ ALI MESHKINI, MD ${ }^{1}$ BOHLOOL HABIBI, ASL PHARM D ${ }^{1}$ FIROOZ \\ SALEHPOUR, MD ${ }^{1}$ EBRAHIM RAFEI, MD,${ }^{1}$ WOURIA FATHI, MD ${ }^{1}$ SEYED HAMED NASERI ALAVI, \\ MD ${ }^{2}$ ALIREZA MAJDI, MD,${ }^{3}$ SEPIDEH RAHIGH AGHASAN, PharmD,${ }^{4}$ SEYED AHMAD NASERI ALAVI, MD ${ }^{1}$ \\ ${ }^{I}$ Department of Neurosurgery, Faculty of Medicine, Tabriz University of Medical Sciences, Tabriz, Iran, ${ }^{2}$ Faculty of Medicine, Guilan University of Medical \\ Sciences, Rasht, Iran, ${ }^{3}$ Neuroscience Research Center, Tabriz University of Medical Sciences, Tabriz, Iran, ${ }^{4}$ Department of Clinical Pharmacy, Faculty of \\ Pharmacy, Tabriz University of Medical Sciences, Tabriz, Iran
}

\begin{abstract}
Background: Guidelines do not suggest the routine use of methylprednisolone (MP) in patients with acute traumatic spinal cord injury (SCI). We tested the hypothesis regarding whether combination therapy with ceftriaxone and MP is superior to MP monotherapy in patients with acute traumatic SCI.

Methods: In a randomized, triple-blind clinical trial, 60 patients with acute (first 8 hours of the injury) traumatic SCI were enrolled at the Tabriz University of Medical Sciences, Tabriz, Iran, between December 2016 and June 2017. Accordingly, the patients were randomly divided into 2 case and control groups $(\mathrm{n}=30$ each). Upon admission, all included patients received a bolus dose of MP at $33 \mathrm{mg} / \mathrm{kg}$ intravenously (IV) for 15 minutes. Then, after 45 minutes, MP infusion was continued for 24 to 48 hours at a $5.4 \mathrm{mg} / \mathrm{kg}$ IV dose. The case group received an additional dose of ceftriaxone at $1 \mathrm{~g} 2$ times a day for 7 days through an IV route. Erythrocyte sedimentation rate (ESR) and C-reactive protein (CRP) were checked and compared between case and control groups upon admission and on the fourth and eighth days. Also, sensory and motor functions were evaluated according to the American Spinal Injury Association (ASIA) grading score upon admission, on the third and seventh days, upon discharge and 6 months after admission.

Results: Analyses showed a significant statistical difference between groups in the changes in CRP levels during days 1 and $4(P=.001)$ and also during days 4 and $8(P=.001)$. However, no significant statistical difference was detected in ESR levels changes between groups during days 1 and $4(P=.073)$, and during days 4 and $8(P=.069)$. ASIA scale was found to be significantly different between the MP plus ceftriaxone group and MP monotherapy upon admission and 6 months after treatment $(P=.001$ for both comparisons). However, the number of variations in the ASIA score had no significant statistical difference between groups 6 months after intervention $(P=.465)$.

Conclusion: The addition of ceftriaxone to the routine therapeutic protocol of acute SCI is accompanied by improved inflammation markers and functional outcomes 6 months after the intervention.
\end{abstract}

Lumbar Spine

Keywords: acute spinal cord injury, methylprednisolone, ceftriaxone, functional outcome, inflammation markers

\section{INTRODUCTION}

The incidence of spinal cord injury (SCI) has grown in recent decades around the world. It constitutes 10,000 new cases of disability and paralysis per year. ${ }^{1,2}$ SCI can be categorized into 2 early and late phases. The early-phase results from the direct physical injury and also local vascular damage leading to microvascular hemorrhage in white and gray matters. The late phase is caused by a severe inflammatory response, vascular distribu- tion changes, change in ionic balances, ion-dependent channels and neurotransmitters, oxidative injury, ischemic-reperfusion injury, excitotoxicity, and cell death. ${ }^{2,3}$

Among these mechanisms, glutamate-induced excitotoxicity is important. Glutamate is an excitatory neurotransmitter that increases in SCI in response to ion-dependent channel damage and is responsible for $\alpha$-amino-3-hydroxy-5-methyl-4 isoxazole propionic acid (AMPA) receptor-related oligodendrocyte injury. ${ }^{1-3}$ However, glutamate 
transporter 1 (GLT-1) clears glutamate from mammalian cells and decreases additional injury to damaged tissue, distribution of injury and inflammatory response. ${ }^{4-6}$

Ceftriaxone, as a member of the cephalosporins family, has been shown to upregulate GLT-1 expression after trauma in the central nervous system and peripheral neuronal cells. ${ }^{1,7}$ The positive effects of ceftriaxone on neuronal cells and recovery in rat models with SCI have been also demonstrated. ${ }^{8}$ Ceftriaxone has been also found to render neuroprotective effects in patients with amyotrophic lateral sclerosis, Huntington disease, spinal muscular atrophy, ${ }^{9}$ and stroke. ${ }^{10}$

Methylprednisolone (MP), as a steroid, is the only approved drug for the treatment of SCI. MP reduces inflammation and improves functional recovery of the spinal cord following acute SCI. ${ }^{11,12}$ However, there is an ongoing debate regarding the appropriate doses needed for patients with acute SCI. ${ }^{13}$ Also, pooled evidence emerging from meta-analyses of existing trials does not support the long-term use of MP in patients with acute SCI, because not only does its chronic use not add therapeutic benefit to these patients, it is associated with an increased risk of gastrointestinal bleeding. ${ }^{14}$

Here, we aimed to test the hypothesis regarding whether combination therapy with ceftriaxone and MP is superior to MP monotherapy in patients with acute traumatic SCI.

\section{PATIENTS AND METHODS}

\section{Study Design}

In a randomized, triple-blind clinical trial, 60 patients with acute (first 8 hours of the injury) SCI were enrolled to assess the effects of ceftriaxone and MP combination therapy versus MP monotherapy. The study was performed at the Imam Reza Educational, Medical and Research Hospital of Tabriz University of Medical Sciences (TUOMS), Tabriz, Iran, between December 2016 and June 2017.

\section{Inclusion and Exclusion Criteria}

Written informed consent was a mandatory criterion for participation in this study. Accordingly, patients between 18 and 60 years old, with Glasgow Coma Scale $\geq 13$, spinal cord injury between the $T_{10}$ and $L_{2}$ levels, and American Spinal
Injury Association (ASIA) grading score between A and D (motor- or sensory-level deficit), were included in this study. On the other hand, patients with head trauma, Glasgow Coma Scale $<13$, sensory and motor deficit score of $\mathrm{E}$ in the ASIA grading system, or a history of drug hypersensitivity, especially to penicillin, ceftriaxone, or other cephalosporins, were excluded from this study. Also, patients who received calcium or related products, had an injury to levels of spine other than $T_{10}$ to $L_{2}$, were pregnant or in the lactation period, or had a history of spinal surgery, diabetes mellitus, chronic diseases, and mental disorders were not enrolled in this study.

\section{Randomization}

Numbers were placed inside closed envelopes with identical shapes, sizes, and colors at the nursing station. Accordingly, the head nurse of the department performed the individual's in-person-based sequencing, and the specialist was not aware of the treatments administered. The numbers were divided into 2 case and control groups, and those received by patients matched with the numbers received from www.random.org. The head nurse of the ward, examiner physician, and data analyzer were not aware of the numbers and the medications. All medications were purchased from 1 pharmaceutical company, and no differences existed between the medications, for example, with regard to appearance, color, form, size, and shape.

\section{Study Methods}

Sixty patients with acute traumatic SCI and ASIA grading system-based sensory and motor deficits were included in the study and divided into 2 case and control groups $(n=30$ each). The ASIA score defines the severity of functional impairment in an individual affected by SCI. This scale uses light touch and a pinprick at multiple points on the body to assess sensation and key motions on both sides of the body. This scaling system is composed of 5 grades starting from A and ending with $\mathrm{E}$. The severity of the functional impairment is highest at $\mathrm{A}$ and lowest at E. Demographic data of the patients, including age and sex, were recorded. All patients were thoroughly examined by a senior neurosurgeon to assess the severity of lesion according to the guidelines of the ASIA score. A blood sample was taken from all of the patients before the medical intervention to assess C-reactive protein (CRP) and 


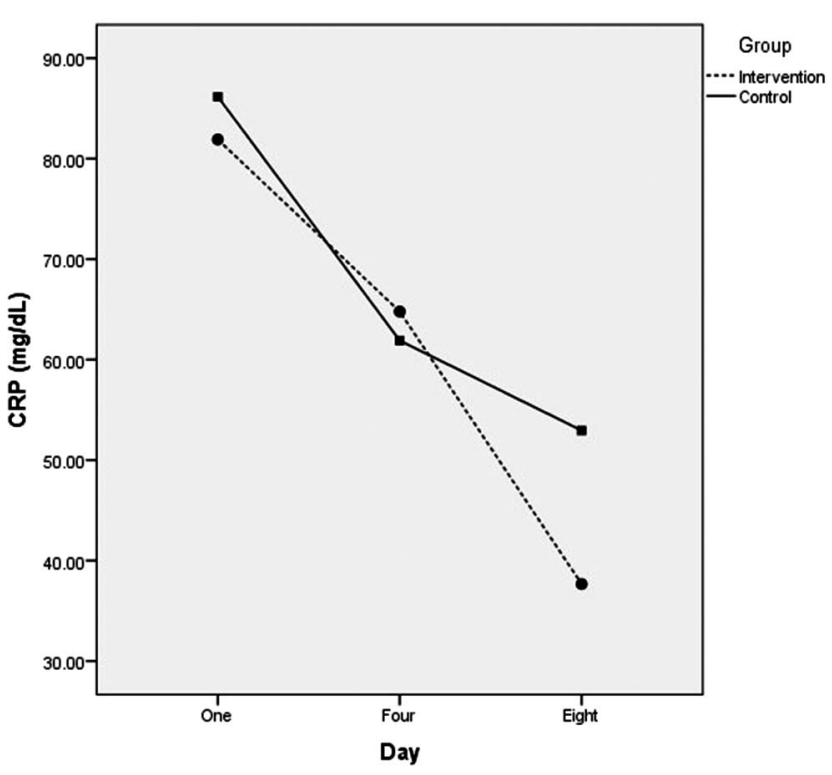

Figure 1. Results of covariance comparison of changes in C-reactive protein (CRP) values between groups. Intervention: ceftriaxone plus methylprednisolone; control: methylprednisolone monotherapy.

erythrocyte sedimentation rate (ESR). Accordingly, and upon admission, all included patients received a bolus of MP at a dose of $33 \mathrm{mg} / \mathrm{kg}$ intravenously (IV) for 15 minutes. Then, after 45 minutes, MP infusion was continued for 24 to 48 hours at a 5.4 $\mathrm{mg} / \mathrm{kg}$ IV dose under the NASCIS 3 protocol. ${ }^{15}$ The case group received an additional dose of ceftriaxone at $1 \mathrm{~g} 2$ times a day for 7 days through the IV route, and the control group received the same dose/ duration/route with normal saline. It should be noted that ceftriaxone and normal saline injections were prepared in a way that made their vials indistinguishable from each other to the personnel and patients. ESR and CRP were checked and compared between case and control groups upon admission and on the fourth and eighth days. Also, sensory and motor functions were evaluated according to the ASIA grading score upon admission, on the third and seventh days, upon discharge and 6 months after admission.

\section{Ethical Considerations}

Written informed consent was obtained from all of the participants in the study or their guardians. The method of the study was approved by the ethics committee of TUOMS under code No. IR.TBZMED.REC.1395.434. Further, the study was approved by the Iranian Clinical Trial Center with reference code No. IRCT2016102713947N5.
Table 1. Mean \pm SD of C-reactive protein (CRP) upon admission, and on the fourth and eighth days after the intervention.

\begin{tabular}{llcc}
\hline Timeline & \multicolumn{1}{c}{ Group } & $\begin{array}{c}\text { CRP, mean } \pm \text { SD, } \\
\text { mg/dL }\end{array}$ & $\boldsymbol{P}$ Value \\
\hline Upon admission & MP + ceftriaxone & $81.90 \pm 13.74$ & .425 \\
& MP & $86.16 \pm 12.32$ & \\
Fourth day & MP + ceftriaxone & $64.78 \pm 13.18$ & .474 \\
Eighth day & MP & $61.90 \pm 11.84$ & \\
& MP + ceftriaxone & $37.66 \pm 14.05$ & .610 \\
& MP & $52.94 \pm 12.47$ & \\
\end{tabular}

Abbreviation: MP, methylprednisolone.

\section{Statistics}

Statistical analyses were conducted using SPSS version 18.0 software (SPSS Inc, Chicago, Illinois). Data were presented as mean \pm standard deviation (SD). Fisher exact test or $\chi^{2}$ test (for categoric variables) and independent samples $t$ test (for numeric data) were used for assessing intergroup differences. Also, repeated measurement design tests and covariance's analysis of variance (ANOVA) were applied for adjustment of abnormal homogeneity. Mann-Whitney $U$ test was used to compare the ASIA grading improvement between admission time and 6 months later. $P<.05$ was considered to be statistically significant.

\section{RESULTS}

\section{Demographic Data}

In this clinical trial, 60 patients with acute traumatic SCI were included and divided into two 30-patient groups (Figure 1). The mean age of the patients was $44.56 \pm 15.65$ years in the case group and $42 \pm 16.28$ years in the control group $(P=.41)$. Of 60 patients, $29(48.3 \%)$ were male and 31 $(51.7 \%)$ were female. No difference existed between groups in terms of $\operatorname{sex}(P=.35)$.

\section{Paraclinical Outcome Measures}

The results showed lower levels of CRP in the combination therapy group compared with the monotherapy group upon admission and on the seventh day, but not on the fourth day. However, the difference in CRP levels between the case and the control groups did not reach a statistical significance in any of the comparisons $(P>.05$ for all comparisons). Further analyses showed a significant statistical difference between groups in the changes of CRP levels during days 1 and $4(P=$ $.001)$ and also during days 4 and $8(P=.001$; Table 1 and Figure 2). 


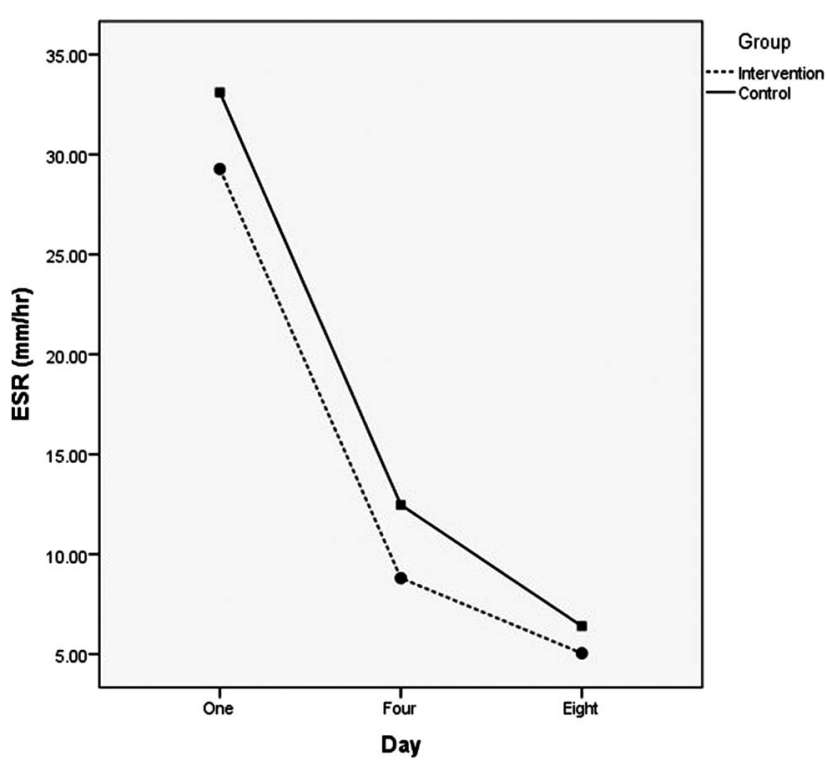

Figure 2. Results of covariance comparison of changes in erythrocyte sedimentation rate (ESR) values between groups. Intervention: ceftriaxone plus methylprednisolone; control: methylprednisolone monotherapy.

We found lower levels of ESR in the combination therapy group compared with the monotherapy group upon admission and on the fourth and seventh days after the intervention. No significant statistical difference existed between the case and control groups in ESR levels on any given day of the assessment. Also, no significant statistical difference was detected in ESR level changes between groups during days 1 and $4(P=.073)$ and days 4 and $8(P=$ .069 ; Table 2 and Figure 3).
Table 2. Mean \pm SD of erythrocyte sedimentation rate (ESR) upon admission, and on the fourth and eighth days after the intervention.

\begin{tabular}{llrc}
\hline Timeline & \multicolumn{1}{c}{ Group } & $\begin{array}{c}\text { ESR, mean } \pm \mathbf{S D}, \\
\mathbf{m m} / \mathbf{h}\end{array}$ & $\boldsymbol{P}$ Value \\
\hline Upon admission & MP + ceftriaxone & $29.27 \pm 5.91$ & .457 \\
& MP & $33.10 \pm 5.37$ & \\
Fourth day & MP + ceftriaxone & $8.80 \pm 3.82$ & .289 \\
& MP & $12.36 \pm 3.34$ & \\
Eighth day & MP + ceftriaxone & $5.04 \pm 2.93$ & .477 \\
& MP & $6.40 \pm 3.25$ & \\
\hline
\end{tabular}

Abbreviation: MP, methylprednisolone.

\section{Clinical Outcome Measures}

We found that grade $\mathrm{B}$ in the ASIA grading system had the highest frequency in both the case and control groups upon admission $(\mathrm{n}=16[26.7 \%]$ and $n=15$ [25\%], respectively). We detected several shifts between different grades in both of the groups (Figure 4). ASIA scale was found to be significantly different between the MP plus ceftriaxone group and MP monotherapy upon admission and 6 months after treatment $(P=.001$ for both comparisons). The differences were found to be negligible upon discharge, on days 3 and 7 after the intervention. Also, the number of variations in the ASIA score was not significantly different between groups 6 months after intervention $(P=.465$; Table $3)$.

\section{DISCUSSION}

Patients who experience acute traumatic SCI commonly have severely impaired or loss of function and diminished quality of life. Accordingly,

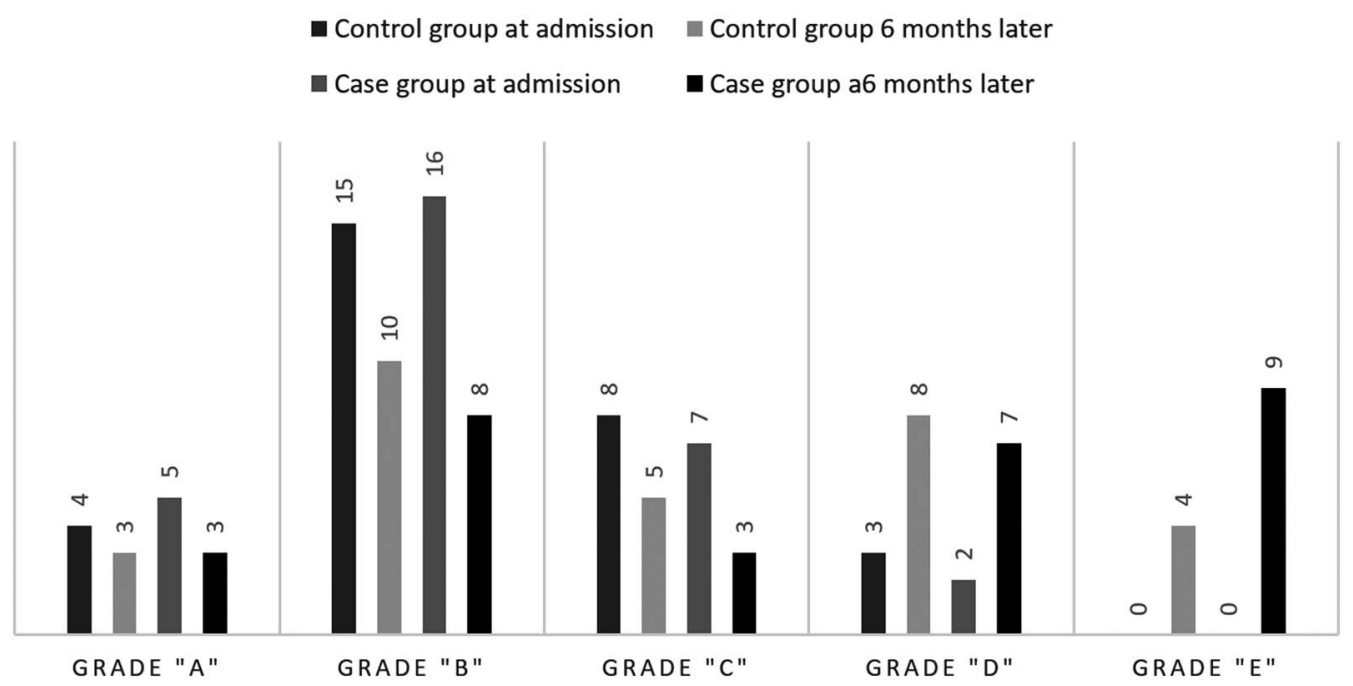

Figure 3. The difference between improvement of the case and control groups based on the American Spinal Injury Association (ASIA) grading system. 


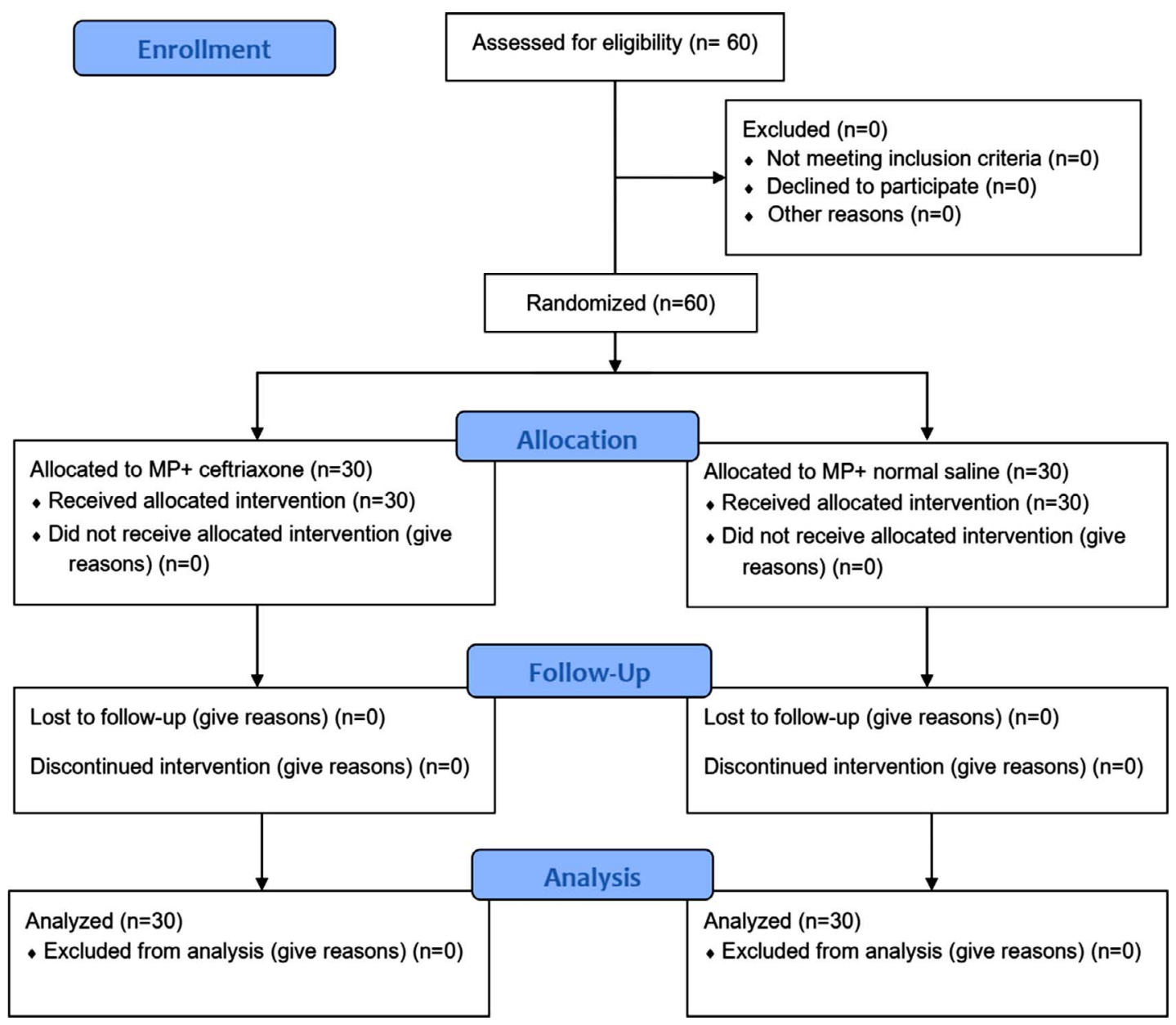

Figure 4. Flowchart of the clinical trial. The style was adopted from Moher D, Schulz KF, Altman DG. The CONSORT statement: revised recommendations for improving the quality of reports of parallel-group randomized trials. Ann Intern Med. 2001;134:657-662.

Table 3. Comparison of the American Spinal Injury Association (ASIA) grading results upon admission and 6 months after discharge between the combination therapy and monotherapy groups.

\begin{tabular}{|c|c|c|c|c|c|c|}
\hline & \multicolumn{5}{|c|}{6 mo After Discharge, No. (\% of All Patients) } & \multirow[b]{2}{*}{ Total } \\
\hline & A & B & $\mathbf{C}$ & D & $\mathbf{E}$ & \\
\hline \multicolumn{7}{|c|}{ Upon admission } \\
\hline A & $6(66.7)$ & $3(3.33)$ & 0 & 0 & 0 & $9(15)$ \\
\hline B & 0 & $15(48.4)$ & $8(25.8)$ & $8(25.8)$ & 0 & $31(51.7)$ \\
\hline $\mathrm{C}$ & 0 & 0 & 0 & $7(46.7)$ & $8(53.3)$ & $15(25)$ \\
\hline $\mathrm{D}$ & 0 & 0 & 0 & 0 & $5(100)$ & $5(8.3)$ \\
\hline Total & $6(10)$ & $18(30)$ & $8(13.3)$ & $15(25)$ & $13(21.7)$ & $60(100)$ \\
\hline \multicolumn{7}{|c|}{6 mo after discharge (intervention group) } \\
\hline A & $3(60)$ & $2(40)$ & 0 & 0 & 0 & $5(16.7)$ \\
\hline B & 0 & $6(37.5)$ & $3(18.8)$ & $7(43.8)$ & 0 & $16(53.3)$ \\
\hline $\mathrm{C}$ & 0 & 0 & 0 & 0 & $7(100)$ & $7(23.3)$ \\
\hline $\mathrm{D}$ & 0 & 0 & 0 & 0 & $2(100)$ & $2(6.7)$ \\
\hline Total & $3(10)$ & $8(26.7)$ & $3(10)$ & $7(23.3)$ & $9(30)$ & $30(100)$ \\
\hline \multicolumn{7}{|c|}{6 mo after discharge (control group) } \\
\hline A & $3(75)$ & $1(25)$ & 0 & 0 & 0 & $4(13.3)$ \\
\hline B & 0 & $9(60)$ & $5(33.3)$ & $1(6.7)$ & 0 & $15(50)$ \\
\hline C & 0 & 0 & 0 & 7 (87.5) & $1(12.5)$ & $8(26.7)$ \\
\hline D & 0 & 0 & 0 & 0 & $3(100)$ & $3(10)$ \\
\hline Total & $3(10)$ & $10(33.3)$ & $5(16.7)$ & $8(26.7)$ & $3(13.3)$ & $30(100)$ \\
\hline
\end{tabular}

the development of novel therapeutic options to enhance sensory and motor recovery is of importance. ${ }^{14}$ Several treatments have been used in patients with acute SCI to alleviate symptoms and improve prognosis. Among these medications, MP is the only conformed treatment routinely used in patients with acute traumatic SCI. However, results emerging from robust clinical trials indicate a small advantage of MP for motor recovery in these patients when the drug is given in the first 8 hours after injury. ${ }^{16,17}$ Evidence from other studies has shown inconsistent results, leading to a sharp decline in the use of MP in the last decade. ${ }^{18-20} \mathrm{In}$ that light, several guidelines do not suggest the routine application of MP in patients with acute traumatic SCI. ${ }^{21,22}$ Thus, the need for the development of new therapeutic options for the treatment of acute traumatic SCI is felt.

Accordingly, in a randomized, triple-blind clinical trial, 60 patients with acute traumatic SCI were randomly divided into 2 groups $(\mathrm{n}=30)$ of $\mathrm{MP}$ 
alone and MP plus ceftriaxone to compare their effects on blood inflammatory markers (ESR and CRP) and functional recovery measures (ASIA grading score). The addition of ceftriaxone to the routine protocol of acute traumatic SCI was able to lower ESR and CRP numeric levels and improve ASIA grade more than the MP alone group. However, only the changes for CRP and ASIA scores were statistically significant at different time points of the study.

It has been shown that both excitotoxicity and oxidative stress play a major role in the late phase of acute traumatic SCI-induced functional impairment. ${ }^{23,24}$ Also, it has been found that patients with SCI have a higher concentration of CRP and ESR in their blood compared with others. ${ }^{25,26}$ Ceftriaxone is a member of cephalosporin antibiotics that has been shown to possess neuroprotective effects. The neuroprotective impacts of ceftriaxone are thought to be mediated by antiexcitotoxicity and antioxidant mechanisms via an increase in GLT-1 expression and glutamate reuptake. ${ }^{27,28}$ Also, the anti-inflammatory effects of ceftriaxone have been established. ${ }^{28}$

In an animal study performed by Tajkey et $\mathrm{al}^{8}$ it was revealed that intraperitoneal ceftriaxone administration at a dosage of $200 \mathrm{mg} / \mathrm{kg} /$ day for 7 days after SCI enhances functional recovery more effectively than monocytes in the rat model of SCI injury. ${ }^{8}$ However, we were not able to reproduce all of the results of the experimental studies on patients with acute traumatic SCI, meaning that addition of ceftriaxone to the existing protocol was associated with neither a decrease in all of the inflammatory markers nor an improvement in ASIA score at all time points of the study.

The difference between the results of experimental studies and our study may arise from several potential explanations. First, animal models are not able to mimic all of the features of human disease. Second, experimental studies may have low internal and external validities, making it difficult to reproduce their results and also generalize them to clinical trials. On the other hand, clinical trials may also have flaws that lower their robustness, such as lack of randomization, allocation concealment, and outcome assessment concealment. ${ }^{29}$ Our study had some limitations, which may have led to the existing results. First, our study was performed on a limited number of patients, and no a priori analysis was performed to evaluate the sample size and power of the study. Thus, it is hard to draw firm conclusions on cohort outcomes and subgroup variations based on this study. Accordingly, studies with a higher number of patients may have more reliable results. Second, we did not assess the visual analog scale in our patients, which is an important measure for acute and chronic pain and thus functional recovery. The score should be measured in future studies. Also, other outcome scores would be useful in future studies (eg, disability scores, quality of life scores, etc).

To the best of our knowledge, this was the first clinical trial set to assess and compare the effects of the addition of ceftriaxone to MP on blood inflammatory factors and functional recovery in patients with acute traumatic SCI. Although the results showed a further decrease in ESR and CRP levels and higher improvement levels after 6 months of injury in the ceftriaxone plus MP group, the differences in ASIA scores were not statistically significant. Future studies should aim at setting placebo-controlled trials with a higher number of patients to validate the findings of this study.

\section{REFERENCES}

1. Tajkey J, Ramazani A, Biglari A, Mazlomzadeh S, Habibi Asl B. Ceftriaxone improves neuron protection and functional recovery in rat model of spinal cord injury. Ann Res Rev Biol. 2014;4(12):1958-1967.

2. Aghazadeh J, Samadi Motlagh P, Salehpour F, et al. Effects of atorvastatin in patients with acute spinal cord injury. Asian Spine J. 2017;11(6):903-907. https://doi.org/10.4184/asj. 2017.11.6.903

3. Meshkini A, Salehpour F, Aghazadeh J, Mirzaei F, Naseri Alavi SA. Riluzole can improve sensory and motor function in patients with acute spinal cord injury. Asian J Neurosurg. 2018;13:656-659.

4. Rothstein JD, Patel S, Regan MR, et al. Beta-lactam antibiotics offer neuroprotection by increasing glutamate transporter expression. Nature. 2005;433(7021):73-77. DOI: 10.1038 /nature 03180

5. Ramos KM, Lewis MT, Morgan KN, et al. Spinal upregulation of glutamate transporter GLT-1 by ceftriaxone: therapeutic efficacy in a range of experimental nervous system disorders. Neuroscience. 2010;169(4):1888-1900. DOI: 10.1016 j.neuroscience.2010.06.014

6. Goodrich GS, Kabakov AY, Hameed MQ, Dhamne SC, Rosenberg PA, Rotenberg A. Ceftriaxone treatment after traumatic brain injury restores expression of the glutamate transporter, GLT-1, reduces regional gliosis, and reduces posttraumatic seizures in the rat. J Neurotrauma. 2013:30:1434 1441. DOI: $10.1089 /$ neu.2012.2712

7. Ramos KM, Lewis MT, Morgan KN, et al. Spinal upregulation of glutamate transporter GLT-1 by ceftriaxone: therapeutic efficacy in a range of experimental nervous system 
disorders. Neuroscience. 2010;169(4):1888-1900. DOI: 10.1016/ j.neuroscience.2010.06.014

8. Tajkey J, Biglari A, Habibi Asl B, Ramezani A, Mazlomzadeh S. Comparative study on the effects of ceftriaxone and monocytes on recovery after spinal cord injury in rat. Adv Pharm Bull. 2015;5(2):189-194. DOI: 10.15171/apb.2015. 026

9. Rothstein JD, Patel S, Regan MR, et al. beta-Lactam antibiotics offer neuroprotection by increasing glutamate transporter expression. Nature. 2005;433(7021):73-77.

10. Chu K, Lee ST, Sinn DI, et al. Pharmacological induction of ischemic tolerance by glutamate transporter-1 (EAAT2) upregulation. Stroke. 2007;38(1):177-182.

11. Chikuda H, Yasunaga H, Takeshita K, et al. Mortality and morbidity after high-dose methylprednisolone treatment in patients with acute cervical spinal cord injury: a propensitymatched analysis using a nationwide administrative database. Emerg Med J. 2014;31(3):201-206.

12. Hall ED, Braughler JM. Effects of intravenous methylprednisolone on spinal cord lipid peroxidation and $(\mathrm{Na}++\mathrm{K}+)$-ATPase activity: dose-response analysis during 1st hour after contusion injury in the cat. $J$ Neurosurg. 1982;57(2):247-253.

13. Cabrera-Aldana EE, Ruelas F, Aranda C, et al. Methylprednisolone administration following spinal cord injury reduces aquaporin 4 expression and exacerbates edema. Mediators Inflamm. 2017;2017:4792932. https://doi.org/10. $1155 / 2017 / 4792932$

14. Evaniew N, Belley-Côté EP, Fallah N, Noonan VK, Rivers CS, Dvorak MF. Methylprednisolone for the treatment of patients with acute spinal cord injuries: a systematic review and meta-analysis. J Neurotrauma. 2016;33(5):468-481. doi: 10. 1089/neu.2015.4192

15. Cheung V, Hoshide R, Bansal V, Kasper E, Chen CC. Methylprednisolone in the management of spinal cord injuries: lessons from randomized, controlled trials. Surg Neurol Int. 2015;6:142. doi: 10.4103/2152-7806.163452

16. Bertrand S, Lacaille JC. Unitary synaptic currents between lacunosum-moleculare interneurones and pyramidal cells in rat hippocampus. J Physiol. 2001;532(pt 2):369-384. doi: 10.1111/j.1469-7793.2001.0369

17. Matsumoto S, Okabe Y, Setoyama H, et al. Inflammatory bowel disease-like enteritis and caecitis in a senescence accelerated mouse P1/Yit strain. Gut. 1998;43(1):71-78. doi: 10. 1136/gut.43.1.71

18. Bracken MB. Steroids for acute spinal cord injury. Cochrane Database Syst Rev. 2012;18(1).

19. Schroeder GD, Kwon BK, Eck JC, Savage JW, Hsu WK, Patel AA. Survey of cervical spine research society members on the use of high-dose steroids for acute spinal cord injuries. Spine (Phila Pa 1976). 2014;39(12):971-977.

20. Druschel C, Schaser KD, Schwab JM. Current practice of methyprednisiolone administration for acute spinal cord injury in Germany: national survey. Spine (Phila Pa 1976). 2013;38(11):669-677.

21. Hurlbert RJ, Hadley MN, Walters BC, et al. Pharmacological therapy for acute spinal cord injury. Neurosurgery. 2013;72(2):93-105.
22. Walters BC, Hadley MN, Hurlbert RJ, et al. Guidelines for the management of acute cervical spine and spinal cord injuries: 2013 update. Neurosurgery. 2013;60(suppl 1):82-91. https://doi.org/10.1227/01.neu.0000430319.32247.7f

23. Nallasamy P, Zhu H, Misra HP, Li Y, Jia Z. Reactive oxygen species and oxidative stress in spinal cord injuryupdated experimental and clinical evidence. In: Laher I, ed. Systems Biology of Free Radicals and Antioxidants. Berlin; Springer; 2014.

24. Park E, Velumian AA, Fehlings MG. Mechanisms of spinal cord injury: a review with an emphasis on the implications for white matter degeneration. J Neurotrauma. 2004;21(6):754-774. http://doi.org/10.1089/0897715041269641

25. Wang TD, Wang YH, Huang TS, Su TC, Pan SL, Chen SY. Circulating levels of markers of inflammation and endothelial activation are increased in men with chronic spinal cord injury. J Formos Med Assoc. 2007;106(11):919-928. DOI: 10.1016/S0929-6646(08)60062-5

26. Estrores IM, Harrington A, Banovac K. C-reactive protein and erythrocyte sedimentation rate in patients with heterotopic ossification after spinal cord injury. J Spinal Cord Med. 2004;27(5):434-437. DOI: 10.1080/10790268.2004. 11752233

27. Tikhonova MA, Ho SC, Akopyan AA, et al. Neuroprotective effects of ceftriaxone treatment on cognitive and neuronal deficits in rat model of accelerated senescence. Behav Brain Res. 2017;14:8-16.

28. Tai $\mathrm{CH}$, Bellesi $\mathrm{M}$, Chen AC, et al. A new avenue for treating neuronal diseases: ceftriaxone, an old antibiotic demonstrating behavioral neuronal effects. Behav Brain Res. 2019;364:149-156. https://doi.org/10.1016/j.bbr.2019.02.020

29. Sadigh-Eteghad S, Majdi A, McCann SK, Mahmoudi J, Vafaee MS, Macleod MR. Correction: D-galactose-induced brain ageing model: a systematic review and meta-analysis on cognitive outcomes and oxidative stress indices. PLoS One. 2017;12(12):e0190328. https://doi.org/10.1371/journal.pone. 0190328

Disclosures and COI: The authors received no funding for this study and report no conflicts of interest.

Corresponding Author: Seyed Ahmad Naseri Alavi, MD, Department of Neurosurgery, Faculty of Medicine, Tabriz University of Medical Sciences, Tabriz, Iran. Phone: (98) 9116137353; Email: dr. arsalan2010@gmail.com and anaseri@tbzmed.ac.ir.

Published 11 November 2020

This manuscript is generously published free of charge by ISASS, the International Society for the Advancement of Spine Surgery. Copyright (c) 2020 ISASS. To see more or order reprints or permissions, see http://ijssurgery.com. 